\title{
Metal island film based structures for sensing using spectrophotometry and ellipsometry
}

\author{
V. Janicki ${ }^{1,2}$, J. Sancho-Parramon ${ }^{1,2}$, S. Bosch ${ }^{2,}$ H. Zorc ${ }^{1}$, F. J. Belarre ${ }^{3}$ and J. Arbiol ${ }^{3,4}$ \\ ${ }^{1}$ Ruđer Bošković Institute, Bijenička 54, 10000, Zagreb, Croatia \\ ${ }^{2}$ Applied Physics and Optics Department, University of Barcelona, Martí i Franques 1, 08028 Barcelona, Spain \\ ${ }^{3}$ Institut de Ciència de Materials de Barcelona, ICMAB-CSIC, Campus de la UAB, 08193 Bellaterra, Catalonia, Spain. \\ ${ }^{4}$ Institució Catalana de Recerca i Estudis Avançats (ICREA), 08010 Barcelona, Catalonia, Spain. \\ *corresponding author, E-mail: janicki@irb.hr
}

\begin{abstract}
Metal island films (MIF) are good candidates for sensors due to the strong sensitivity of the localized surface plasmon resonance (SPR) to the environment refractive index. The strong near field enhancement in the vicinity of the island surface can be even higher if a metal layer (ML) is placed close to a MIF. Structures containing MIF with and without ML are prepared and sensitivities of spectrophotometric and ellipsometric features of the measurements compared. It is shown that simple MIF is preferable for ellipsometry-based sensing and the one including $\mathrm{ML}$ in the case of spectrophotometric measurements.
\end{abstract}

\section{Introduction}

Metal island films, consisting of two-dimensional ensembles of metal particles deposited on a substrate, have strong potential for plasmonic biosensing. SPR of isolated particles is highly sensitive to refractive index changes of the environment, enabling to detect presence of different analytes [1-2]. MIFs are attractive candidates for the production of performance- and cost-efficient sensors. For this reason, sensing using MIF is typically based on shift of the maximum of the SPR band upon changes of dielectric environment. In addition, the strong near field enhancement taking place close to the island surface, allows amplification of Raman signal [3-6] or infrared absorption [7-10] of closely located molecules. The near field enhancement is largest for very small particles and inter-particle distances, which are typically few nanometers in the case of MIF. Strong coupling between SPR of metal nanoparticles and propagating surface plasmon of a nearby metal film (polariton), enhances this electric near field largely [11-13]. This work compares the sensitivity of MIF-based plasmonic structures, consisting of a single MIF layer or including a compact ML nearby MIF, to the quantity of dielectric material surrounding the metal nanoparticles. The sensitivity is studied for the case of spectrophotometric and ellipsometric measurements.

\section{Experimental}

The samples were prepared by electron beam evaporation in a modified Varian 3117 chamber. The quartz substrates were positioned onto a rotating calotte to ensure a uniform thickness of the layers over the sample surface. Base pressure was $6 \cdot 10^{-6}$ Torr. Prior to the deposition of $\mathrm{Au}$ island film the samples were preheated to $250^{\circ} \mathrm{C}$ to enhance metal island growth [14, 15]. Mass thickness of the layers was controlled by a quartz crystal monitor. Deposition rates were $10 \AA /$ s for $\mathrm{SiO}_{2}$ and $1 \AA / \mathrm{s}$ for $\mathrm{Au}$.

The first type of sample structure is quartz/MIF/SiO $\mathrm{S}_{2} \quad\left(\mathrm{MIF}^{*}\right.$ serial $)$ and the second quartz/Au/SiO $2 / M I F / \mathrm{SiO}_{2}$ (AuMIF* serial). These samples are designed to test the sensing potential of MIF, as they would simulate the effect of adsorbed analyte layers at MIF. The samples within one serial differ in the mass thickness of capping $\mathrm{SiO}_{2}$ layer $\left(\mathrm{d}_{\text {capp }}\right)$, that was 3, 6, 9 and $12 \mathrm{~nm}$. Additional samples having structure quartz/MIF, quartz/Au, quartz/Au/ $/ \mathrm{SiO}_{2}$ and quartz/Au/SiO$/ \mathrm{MIF}$ are deposited as well. The mass thickness of compact Au ML is $70 \mathrm{~nm}$ and of the spacer $\mathrm{SiO}_{2}$ layer between ML and MIF is $12 \mathrm{~nm}$. Mass thickness of $\mathrm{Au}$ for MIF is $7 \mathrm{~nm}$. MIF layer is prepared in the same deposition process for all the samples. Also, $\mathrm{SiO}_{2}$ capping layers for both serials are prepared in the same deposition process. Therefore, their thicknesses and optical parameters in both structures are assumed to be the same.

One more sample with $7 \mathrm{~nm} \mathrm{Au} \mathrm{MIF} \mathrm{and} 9 \mathrm{~nm}$ $\mathrm{SiO}_{2}$ capping layer is prepared on a $\mathrm{Si}$ substrate covered with $10 \mathrm{~nm}$ of predeposited $\mathrm{SiO}_{2}$. It is investigated by transmission electron microscopy (TEM), to verify the island-growth and the coverage of the islands with the capping layer. This sample is prepared as a cross-section, firstly grinded and finally ion milled. In order to obtain the average grain size and nanoparticle morphology Bright field (BF) transmission electron microscopy (TEM) images are done in a Jeol 2010F field emission gun microscope operated at $200 \mathrm{KV}$. In order to differentiate between $\mathrm{Si}$ and $\mathrm{SiO}_{2}$ and achieve clear composition images, energy filtered TEM (EFTEM) is made $[16,17]$.

Spectroscopic (reflectance $R$ and transmittance $T$ ) and ellipsometric measurements of the two series of samples are analysed. $R$ and $T$ are measured in the range 300-1100 nm at Perkin Elmer Lambda 25 spectrometer. Ellipsometric functions $\Psi$ and $\Delta$ are measured in the range 4.35-0.57 eV (that corresponds to 285-2175 nm), at incidence angles $25^{\circ}, 45^{\circ}, 55^{\circ}, 65^{\circ}$ and $80^{\circ}$ at Woollam VASE Ellipsometer. Additionally, $T$ is measured at 
ellipsometer in the same range as $\Psi$ and $\Delta$, at normal incidence.

\section{Modelling and characterization}

In a previous study it has been shown that Au metal island films deposited in the same conditions as here have dimensions that are much smaller than the wavelength of the performed measurements [14]. For this reason it is not expected significant scattering. Therefore, in the analysis of the spectral characteristics, absorptance $A$ defined as $A=1-T$ $R$ is included as well.

MIF layers of the studied samples contain not only $\mathrm{Au}$ metal islands, but also $\mathrm{SiO}_{2}$ from the capping layer that fills the space between the islands. Fig. 1 shows the TEM cross section of $\mathrm{Au}$ MIF covered with $\mathrm{SiO}_{2}$. The mean grain height of the $\mathrm{Au}$ nanoparticles is approximately $14 \mathrm{~nm}$. However, the size distribution is wide and grains from 12 to $17 \mathrm{~nm}$ are present. There is enough mass thickness of this capping layer to fill the space between the islands and make an over layer.

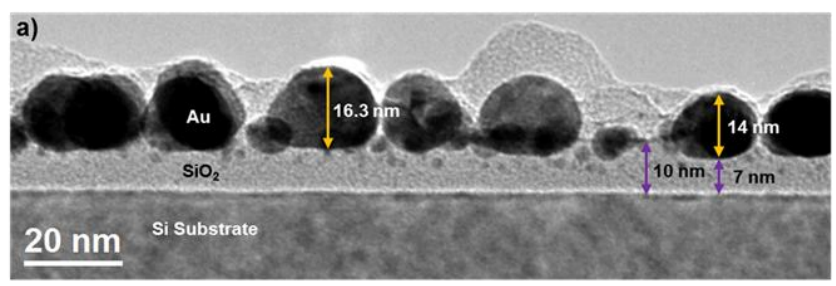

Figure 1. Cross-section, bright-field TEM image of the Au nanoparticles covered with $9 \mathrm{~nm}$ mass thickness $\mathrm{SiO}_{2}$ capping layer.

First, the samples having structure quartz/Au, and quartz/Au/SiO 2 are analyzed. The obtained optical constants and thicknesses of $\mathrm{Au}$ layer and $\mathrm{SiO}_{2}$ under layer are used as known parameters in modelling of the rest of the samples. In order to obtain thicknesses and optical parameters of the layers, the measurements are analyzed with the WVASE32 software. Since the optical parameters of MIF are influenced by the parameters of the dielectric environment, it was expected that they would change with $d_{\text {capp. }}$. For this reason the MIF and the capping layer are modelled as one effective layer (see Fig. 2.). Since effective medium theories were shown inadequate to describe dielectric functions of MIF, they are modelled with Gauss oscillators [15, 18]. The optical parameters of dielectric constants (real and imaginary part, $\varepsilon_{1}$ and $\varepsilon_{2}$ ) of this layer are modelled with a sum of 5 Gauss oscillators. Introducing more oscillators does not improve quality of fit significantly. Modelled data fit the experimental data very well (Fig. 3.). Variation of the optical constants with $\mathrm{d}_{\text {capp }}$ of each model was monitored.

The measured optical spectra are analysed in order to determine the maxima or minima with either a significant central wavelength $\left(\lambda_{o}\right)$ shift or change of the intensity $\left(I_{o}\right)$ with $d_{\text {capp. }}$ A linear fit of central wavelength and intensity values as functions of $d_{\text {capp }}$ is done and sensitivity is calculated as $S_{x}=|\mathrm{d} x / \mathrm{d} d|$, where $x$ denotes $\lambda_{o}$ or $I_{o}$ of the spectral features and $d$ is mass thickness of $\mathrm{SiO}_{2}$ deposited above $\mathrm{Au}$ islands. One could expect that for islands completely embedded in $\mathrm{SiO}_{2}$, the change in $\mathrm{d}_{\text {capp }}$ will not result in a significant change of the SPR characteristics and therefore the sensitivity would decrease with capping layer thickness. However, the range of $d_{\text {capp }}$ used in this work is comparable to the vertical dimension of the islands [14]: as seen in Fig. 1, $9 \mathrm{~nm}$ of capping layer resuls in a very thin over layer covering the islands. In this context, it can be justified that the variation of $x$ with $\mathrm{d}_{\text {capp }}$ appears to be linear.

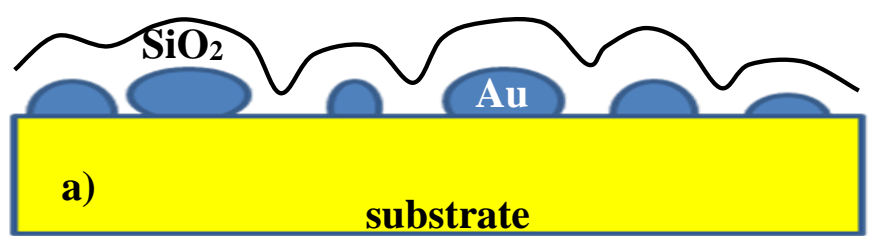

\section{$\mathrm{Au}+\mathrm{SiO}_{2}$}

b)

\section{substrate}

Figure 2. Schematic presentation of a MIF* sample consisting of $\mathrm{Au}$ island film covered with capping $\mathrm{SiO}_{2}$ layer, according to Fig.1 (a) and the corresponding model used in optical characterization (b).
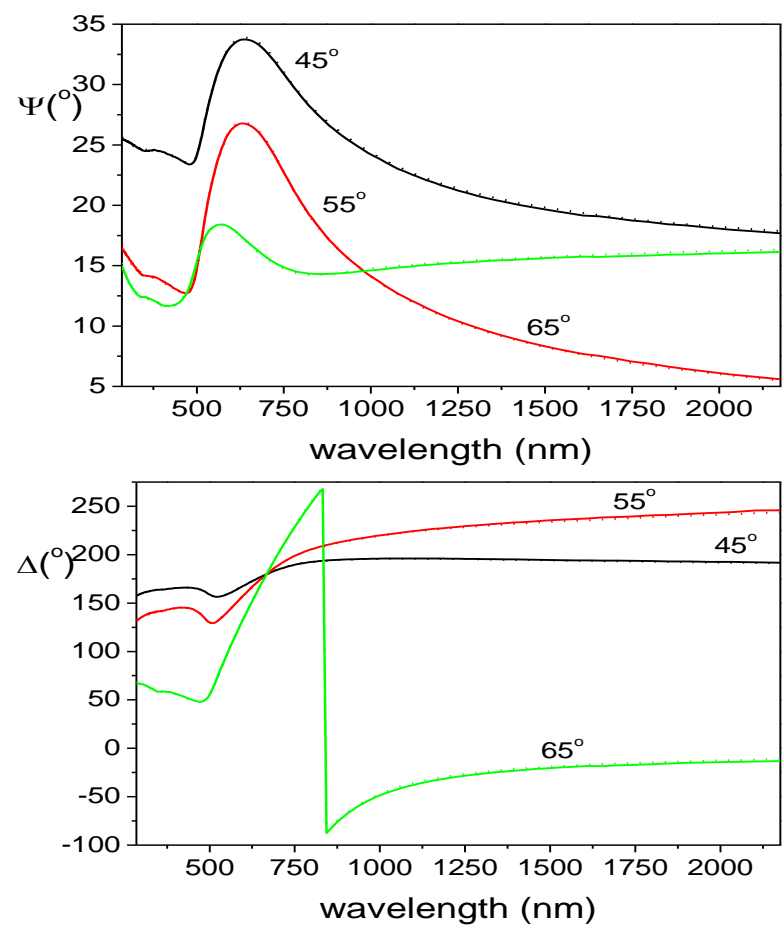

Figure 3. Experimental (full line) and modelled (dots) ellipsometric functions of MIF* serial sample with capping layer $\mathrm{SiO}_{2}$ mass thickness $9 \mathrm{~nm}$. Qualities of fit for all the samples are similar. 


\section{Results and discussion}

The spectra originating from spectrophotometric and ellipsometric measurements are presented in Fig. 4. and Fig. 5. These spectra show that $I_{o}$ and $\lambda_{o}$ of the peaks/dips between 600 and $700 \mathrm{~nm}$ (corresponding to the SPR band, see Fig. 4.) are significantly changing with $d_{\text {capp. }}$. Parameters of these peaks are used for calculation of sensitivities. AuMIF* transmittance spectra show a pronounced dip around $550 \mathrm{~nm}$ that can be related to the coupling of MIF surface plasmon with polariton in compact Au layer [4]. Thus, for the AuMIF*, the peak located above $650 \mathrm{~nm}$, related to SPR, is used for the sensitivity determination. Dielectric constants of the effective layers obtained as the result of optical characterization based on ellipsometric measurements are presented in Fig. 6.
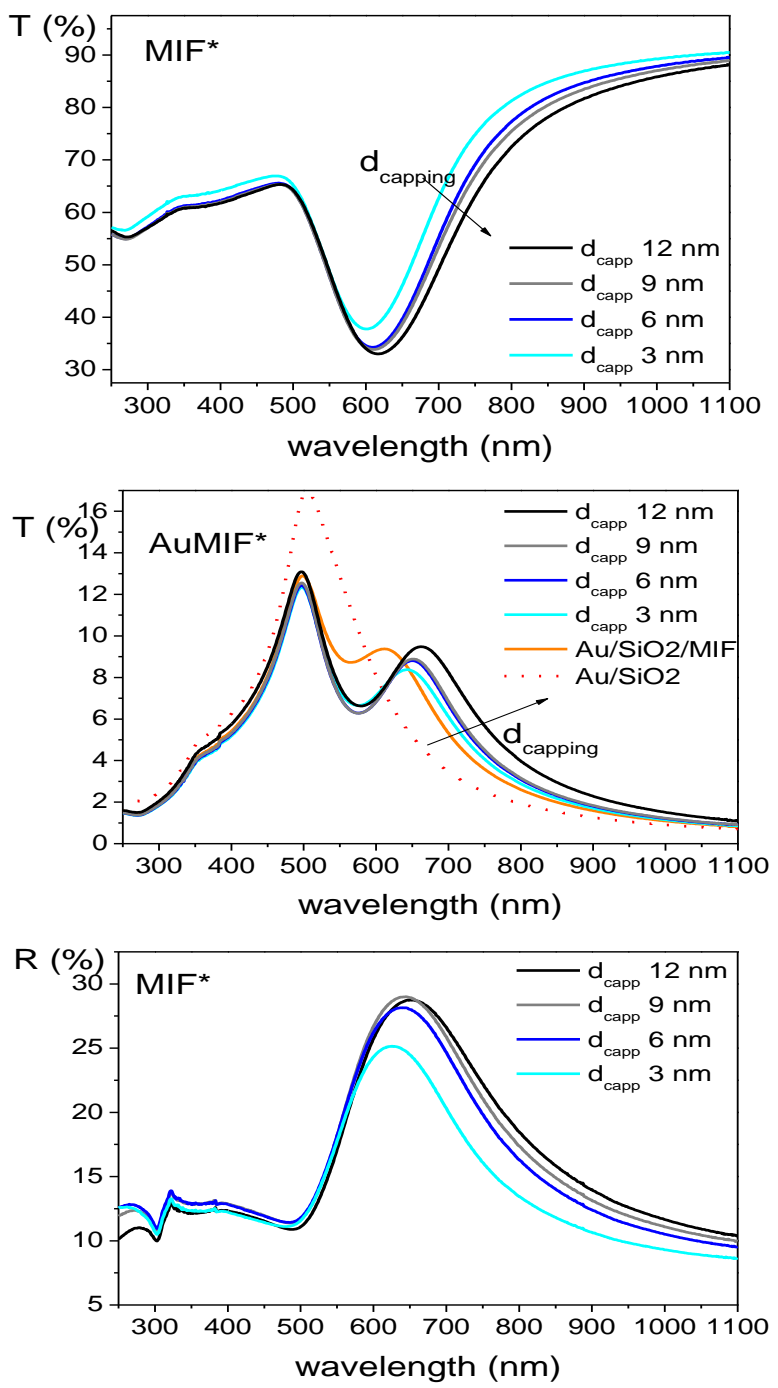


Figure 4. Spectrometric measurements showing characteristical change of spectra due to the increase of $\mathrm{d}_{\text {capp. }}$. Measurements of $\mathrm{Au} / \mathrm{SiO}_{2}$ and $\mathrm{Au} / \mathrm{SiO}_{2} / \mathrm{MIF}$ are presented for comparison.

The calculated sensitivities are presented in Tab.1. and Tab.2. In order to compare intensity sensitivities $S_{I 0}$ of different measurements, they are all normalized to $0-100 \%$ scale. The quantity $I_{c}$, defined as $I_{c}=\sin 2 \Psi \cos \Delta$, is the actual magnitude being measured by the ellipsometer. The changes of $\cos \Delta\left(65^{\circ}\right)$ with $\mathrm{d}_{\text {capp }}$ are presented in Fig. 5 .

The sensitivity of the central wavelength shift $S_{\lambda 0}$ is larger than the sensitivity of the intensity change $S_{I 0}$. This observation agrees with the previous studies showing that changes of the dielectric environment have a stronger effect on the SPR position than on its intensity [4, 14]. In particular, it is found that AuMIF* serial transmittance peak shift sensitivity $S_{\lambda 0}\left(T_{\text {AuMIF*}}\right)$ is higher than the one of $\mathrm{MIF}^{*}$. Since $T$ measurements are simple and widely available, this makes AuMIF* structure more practical for sensing purposes. $S_{\lambda O}\left(A_{\mathrm{AuMIF}}\right)$ also shows higher value than the one of MIF*. However, $S_{\lambda 0}\left(R_{\mathrm{MIF}}\right)$ has the highest sensitivity of all the spectrometric measurements. 

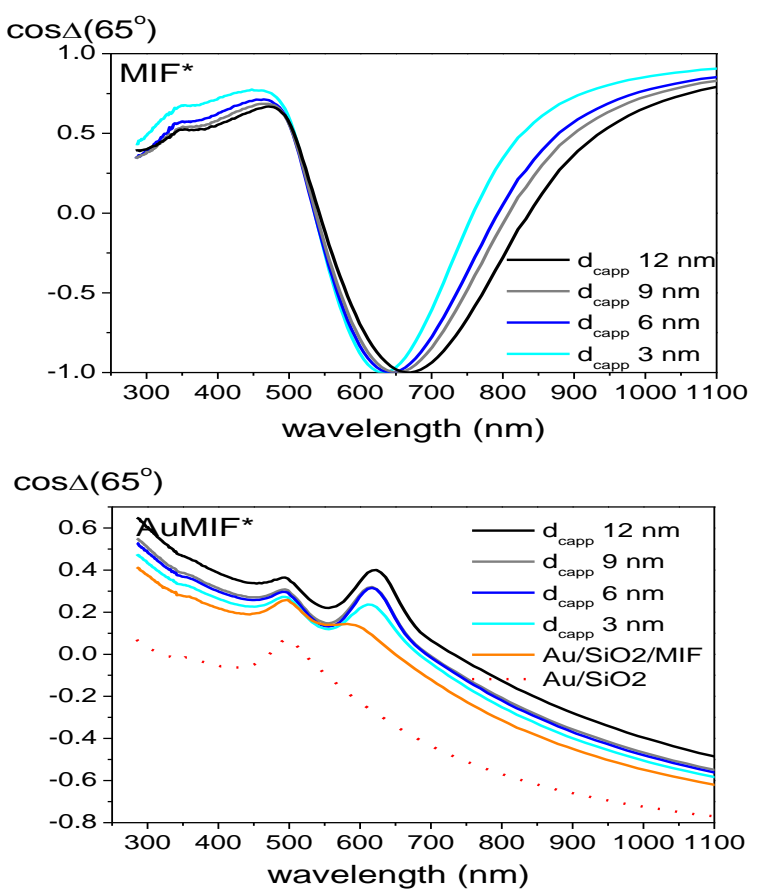

Figure 5. The changes of $\cos \Delta\left(65^{\circ}\right)$ with $\mathrm{d}_{\text {capp }}$.

$S_{I O}\left(T_{\mathrm{MIF} *}\right)$ has higher values than $S_{I 0}\left(\mathrm{~T}_{\mathrm{AuMIF} *}\right)$ and the opposite behaviour is observed for reflectance measurements. This can be explained by the effect of the Au compact metal layer. ML supplies a larger overall reflectivity and lowers transmitivitty with respect to the MIF* structures, hence enhancing or decreasing the associated sensitivities. However, $S_{\lambda O}(R)$ is significantly lower for the AuMIF* serial than for the MIF*. This qualitative difference appears to be related to the excitation of the surface plasmon polariton in the Au film. The SPR of the metals islands does not depend only on the dielectric environment but also on the Au film, hence showing a smaller dependence on $d_{\text {capp }}$. In the case of $S_{I 0}$, the AuMIF* series presents a slightly larger value than the MIF*. In this case one has to take into account that the wavelength refers to the peaks for AuMIF* and to the dips for MIF*. The interplay between the SPR of the islands and the surface plasmon polariton in the ML and its effect in the optical spectra will be the topic of further investigations, supported with numerical simulations. Regarding ellipsometric measurements, $S_{\lambda o}\left(\mathrm{MIF}^{*}\right)$ are significantly higher than for AuMIF*. In the case of $S_{I 0}$ the situation is opposite. However, comparing $S_{\lambda o}$ and $S_{I}$ obtained from ellipsometry, $S_{I}$ values are lower. Dielectric functions $\varepsilon_{1}$ and $\varepsilon_{2}$, obtained from optical characterization based on ellipsometric measurements, give high values of $S_{\lambda o}\left(\mathrm{MIF}^{*}\right)$.

Quantities the most sensitive to spectral shift are shown to be $\cos \Delta\left(65^{\circ}\right)$ and $I_{c}\left(65^{\circ}\right)$, both obtained for ellipsometric measurements of $\mathrm{MIF}^{*}$. High sensitivity of $\Delta$ function in this case originates from the fact that generally it is sensitive to properties of surfaces [19]. It means that it carries the information about the surface of the sample, which corresponds to MIF covered with capping $\mathrm{SiO}_{2}$.
Contrary to this, $R$ and $\Psi$, for example, are dominated with intrinsic properties of the sample [20,21]. Corresponding measurements performed at other angles do not show so high change of $\Delta$ as for $65^{\circ}$. This shows the importance of choosing the angle having the highest sensitivity for the measurements.

High spectral shift sensitivities originate from the expected sensitivity of SPR to the change of the environment. This is confirmed by high values of $S_{\lambda 0}\left(\varepsilon_{1}, \varepsilon_{2}\right)$ of MIF*. On the contrary, spectral shifts of AuMIF* dielectric permittivity are poor. One possible explanation might be that the coupling of localized surface plasmon in MIF and surface polariton in ML is screening the influence of change of environment to SPR. In this case increasing the distance between MIF and ML, by thicker spacer, the coupling should become weaker [11]. Modelling of the studied structures of samples could check for the optimal design of the proposed sensors.

The quality of a plasmonic structure as a refractive index sensor is often quantified with the figure of merit (FOM) that is usually defined as the ratio of the refractive index sensitivity and width of the plasmon resonance. However, the determination of the effective refractive index acting on the islands, that are either partially covered or inhomogeneously surrounded by $\mathrm{SiO}_{2}$, is not a straightforward task since it would require a precise knowledge of the degree of the islands coverage. Therefore, a quantitative comparison of the presented structures quality with, for instance, ordered structures that would present larger FOM due to their narrow SPR spectral width, is not possible. Nevertheless, it is expected that the presented results, i.e., the larger sensitivity offered by certain type of optical measurements for a given sample structure, can be generalised regardless the absolute quality of the sample as refractive index sensor. 

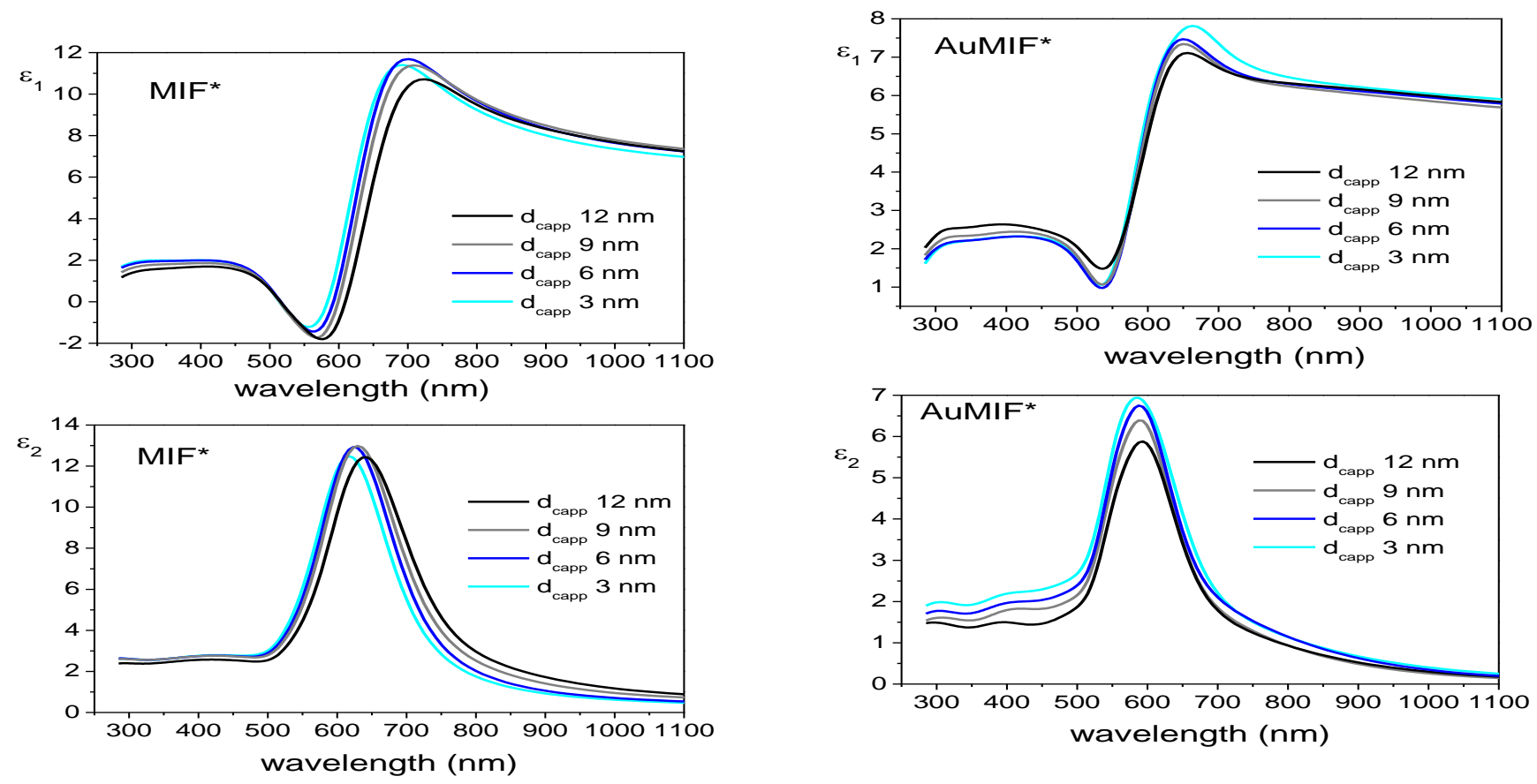

Figure 6. Dielectric functions of effective layers obtained as the result of optical characterization based on ellipsometric measurements.

Table 1: Sensitivities of the measurements

\begin{tabular}{lllll}
\hline & $\mathrm{S}_{\lambda_{0}}$ & \multicolumn{3}{l}{$\mathrm{S}_{\text {Io }}\left(\mathrm{nm}^{-1}\right)$} \\
\cline { 2 - 5 } & $\mathrm{MIF}^{*}$ & AuMIF* $^{*}$ & $\mathrm{MIF}^{*}$ & AuMIF* $^{*}$ \\
\hline $\mathrm{T}$ & 1.67 & $\mathbf{1 . 9 3}$ & $\mathbf{0 . 4 9}$ & 0.11 \\
\hline $\mathrm{R}$ & $\mathbf{2 . 9 3}$ & 1.53 & 0.39 & $\mathbf{0 . 6 3}$ \\
\hline $\mathrm{A}$ & 1.07 & $\mathbf{1 . 4 7}$ & 0.2 & $\mathbf{0 . 5 8}$ \\
\hline$\psi\left(45^{\circ}\right)$ & 1.28 & 1.11 & $<0.1$ & $<0.1$ \\
\hline$\psi\left(80^{\circ}\right)$ & $\mathbf{2 . 8 3}$ & $<0.1$ & $<0.1$ & $<0.1$ \\
\hline $\cos \Delta\left(45^{\circ}\right)$ & 0.58 & $\mathbf{1 . 2 5}$ & 0.16 & $\mathbf{0 . 6 5}$ \\
\hline $\cos \Delta\left(65^{\circ}\right)$ & $\mathbf{4 . 3 1}$ & 1.23 & $<0.1$ & $\mathbf{0 . 8 2}$ \\
\hline $\mathrm{I}_{\mathrm{c}}\left(65^{\circ}\right)$ & $\mathbf{3 . 6 8}$ & 0.47 & 0.34 & $\mathbf{0 . 4 7}$ \\
\hline
\end{tabular}

Table 2: Sensitivities of dielectric permittivities obtained from optical characterization

\begin{tabular}{lllll}
\hline & \multicolumn{2}{c}{$\mathrm{S}_{\lambda_{0}}$} & \multicolumn{2}{c}{$\mathrm{S}_{\text {Io }}\left(\mathrm{nm}^{-1}\right)$} \\
\cline { 2 - 5 } & MIF* $^{*}$ & AuMIF* & MIF* $^{*}$ & AuMIF* \\
\hline$\varepsilon_{1}$ & $\mathbf{2 . 7 7}$ & 0.56 & 0.2 & 0.12 \\
\hline$\varepsilon_{2}$ & $\mathbf{2 . 2 4}$ & $<0.1$ & $<0.1$ & 0.10 \\
\hline
\end{tabular}

\section{Conclusions}

Overall, AuMIF* structure is preferable for sensing in the case of spectrophotometric measurements, while ellipsometric measurements give advantage to MIF* structure having high values of spectral shift sensitivity. These results offer a guideline for design of MIF based sensing structures depending on the availability of optical measurements.

\section{Acknowledgements}

V. Janicki would like to thank European Science Foundation (ESF) for the activity entitled 'New Approaches to Biochemical Sensing with Plasmonic Nanobiophotonics (PLASMON-BIONANOSENSE) Exchange Grant 3853. J. Abriol acknowledges funding from the Spanish MICINN Project MAT2010-15138 and Generalitat de Catalunya (2009 SGR 770). 


\section{References}

[1] A. Vaskevic, I. Rubinstein, Localized Surface Plasmon Resonance Sensing (LSPR) Spectroscopy in Biosensing, Handbok of Biosensors and Biochips, Wiley, 2007.

[2] G. Xu, M. Tazawa, P. Jin, S. Nakao, and K. Yoshimura, Wavelength tuning of surface plasmon resonance using dielectric layers on silver island films, App. Phys. Lett 82, 3811, 2003.

[3] M. Moskovits, Surface-enhanced Raman spectroscopy: a brief retrospective, J. Raman Spectrosc., Vol. 36, No. 36, pp. 485-496, 2005.

[4] M. Fleischmann, P. J. Hendra and A. J. McQuillan, Raman spectra of pyridine adsorbed at a silver electrode, Chem. Phys. Lett. 26 (2) pp. 163-166, 1974.

[5] D. J. Jeanmaire and R. P. van Duyne, Surface Raman electrochemistry. Part I. Heterocyclic, aromatic and aliphatic amines adsorbed on the anodized silver electrode, J. Electroanal. Chem. 84, pp. 1-20, 1977

[6] M. G. Albrecht and J. A. Creighton, Anomalously intense Raman spectra of pyridine at a silver electrode, J. Am. Chem. Soc. 99 (15), pp. 5215-5217, 1977.

[7] M. Osawa, Surface Enhanced Infrared Absorption, Topics Appl. Phys., Vol. 81, pp. 163-187, 2001.

[8] A. Hartstein, J. R. Kirtley, J. C. Tsang, Enhancement in the infrared absorption from molecular monolayers with thin metal overlayers, Phys. Rev. Lett. 45, pp. 201-204, 1980

[9] A. Hatta, T. Ohshima, W. Suetaka, Observation of the enhanced infrared absorption of p-nitrobenzoate on $\mathrm{Ag}$ island films with an ATR technique, Appl. Phys. A 29, pp. 71-75 (1982)

[10] A. Hatta, Y. Suzuki, W. Suetaka, Infrared absorption enhancement of monolayer species on thin evaporated Ag films by use of a Kretschmann configuration, Evidence for two types of enhanced surface electric fields, Appl. Phys. A 35, pp. 135-140, 1984

[11]H. R. Stuart, D. G. Hall, Enhanced dipole-dipole interaction betwen elementary radiators near surface, Phys. Rew. Lett., Vol. 80, pp. 5663-5666, 1998.
[12] Y. Chu, K. B. Crozier, Experimental study of the interaction between localized and propagating surface plasmons, Opt.Lett., Vol. 34, No. 3, pp. 244-246, 2009.

[13]L. C. T. Shoute, Multilayer substrate-mediated tuning resonance of plasmon and SERS EF of nanostructured silver, ChemPhysChem., Vol 11, pp.2539-2545, 2010.

[14] M. Lončarić, J. Sancho-Parramon, M. Pavlović, H. Zorc, P. Dubček, A. Turković, S. Bernstorff, G. Jakopic, A. Haase, Optical and structural characterization of silver island films on glass substrates, Vacuum, Vol. 24, pp. 188-192, 2010.

[15] J. Sancho-Parramon, V. Janicki, H. Zorc, Tuning the effective dielectric function of thin film metal-dielectric composites by controlling the deposition temperature, $J$. Nanophotonics, Vol. 5, pp. 051805-1-8, 2011.

[16] P. Pellegrino, B. Garrido, C. Garcia, J. Arbiol, J.R. Morante, M. Melchiorri, N. Daldosso, L. Pavesi, E. Scheid, G. Sarrabayrouse, J. Appl. Phys., 97 (7), 074312/1-8, 2005.

[17]P. Pellegrino, B. Garrido, J. Arbiol, C. Garcia, Y. Lebour, J.R. Morante, Appl. Phys. Lett., 88 (12), 121915, 2006.

[18] M. Lončarić, J. Sancho-Parramon, H. Zorc, Optical properties of gold island films - a spectroscopic ellipsometry study, Thin Solid Films, Vol. 519, pp. 2946-2950, 2011.

[19]A. V. Tikhonravov, M. K. Trubetskov, A. V. Krasilnikova, E. Masetti, A. Duparre, E. Quesnel, D. Ristau, Investigation of the surface micro-roughness of fluoride films by spectroscopic ellipsometry,Thin Solid Films, Vol. 397, pp. 229-237, 2001.

[20]A. V. Tikhonravov, M. K. Trubetskov, A. V. Krasilnikova, Spectroscopic ellipsometry of slightly inhomogeneous nonabsorbing thin films with arbitrary refractive-index profiles: theoretical study, Appl. Opt. Vol. 37, pp. 5902-5911, 1998.

[21] K. Lodewijks, W. Van Roy, G. Borghs, L. Lagae, P. Van Dorpe, Boosting the figure-of-merit of LSPR-based refractive index sensing by phase-sensitive measurements, Nano Lett., Vol. 12, pp. 1655-1659, 2012. 